\title{
Association of HLA-DR1 with the allergic response to the major mugwort pollen allergen: molecular background
}

Bernhard Knapp 1 , Gottfried Fischer ${ }^{2}$, Dries Van Hemelen ${ }^{3}$, Ingrid Fae ${ }^{2}$, Bernard Maillere ${ }^{4}$, Christof Ebner ${ }^{5}$, Wolfgang Schreiner ${ }^{1}$, Barbara Bohle ${ }^{3,6}$ and Beatrice Jahn-Schmid ${ }^{3 *}$

\begin{abstract}
Background: Mugwort pollen allergens represent the main cause of pollinosis in late summer. The major allergen, Art v 1, contains only one single immunodominant, solely HLA-DR-restricted T cell epitope (Art $\vee 1_{25-36}$ ). The frequency of HLADRB1*01 is highly increased in mugwort-allergic individuals and HLA-DR1 serves as restriction element for Art $\vee 1_{25-36}$. However, Art $\vee 1_{25-36}$ also binds to HLA-DR4 with high affinity and DR1-restricted Art $\vee 1_{25-36}$-specific $T$ cell receptors can be activated by HLA-DR4 molecules. To understand the predominance of HLA-DR1 in mugwort allergy in spite of the degeneracy in HLA/peptide-binding and TCR-recognition, we investigated the molecular background of Art v $1_{25-36}$ /MHC/TCR interactions in the context of HLA-DR1 compared to -DR4.

Results: The majority of Art $\vee 1_{25-36}$-specific $T$ cell lines and clones from HLA-DR1 carrying, mugwort pollen-allergic donors reacted to synthetic and naturally processed Art v 1-peptides when presented by HLA-DR1 or HLA-DR4 expressing antigen presenting cells. However, at limiting peptide concentrations DR1 was more effective in T cell stimulation. In addition, the minimal epitope for $50 \%$ of Art $v 1_{25-36}$-specific T cells was shorter for DR1 than for DR4. In vitro binding assays of Art $v 1_{25-36}$ mutant peptides to isolated DR1- and DR4-molecules indicated similar binding capacities and use of the same register. In silico simulation of Art v $1_{25-36}$ binding to HLA-DR1 and -DR4 suggested similar binding of the central part of the peptide to either molecule, but a higher flexibility of the $\mathrm{N}$ - and C-terminal amino acids and detachment at the C-terminus in HLA-DR1.
\end{abstract}

Conclusions: The predominance of HLA-DR1 in the response to Art $v 1_{25-36}$ may be explained by subtle conformation changes of the peptide bound to DR1 compared to DR4. Computer simulation supported our experimental data by demonstrating differences in peptide mobility within the HLA-DR complex that may influence TCR-binding. We suggest that the minor differences observed in vitro may be more relevant in the microenvironment in vivo, so that only presentation by HLA-DR1, but not -DR4 permits successful T cell activation.

Keywords: HLA association, Peptide/MHC-class II-TCR interaction, T cell recognition, Allergen, Art v 1, Molecular dynamics simulation

\footnotetext{
* Correspondence: beatrice.jahn-schmid@meduniwien.ac.at

${ }^{3}$ Department of Pathophysiology and Allergy Research, Medical University of Vienna, Vienna, Austria

Full list of author information is available at the end of the article
} 


\section{Background}

CD4+ T helper (Th) cells play a major role in the induction and maintenance of IgE-mediated allergy [1], which is characterized by an excessive Th2 response. The Th2cytokines, interleukin-4 and interleukin-13, lead to the production of allergen-specific IgE antibodies which bind to high affinity receptors on effector cells. Upon renewed allergen contact, cross-linking of these IgE antibodies leads to the release of inflammatory mediators, which cause allergic symptoms. Allergen-specific CD4+ Th cells recognize their cognate peptide via their $\mathrm{T}$ cell receptor (TCR) in the context of self major histocompatibility complex (MHC) class II molecules on the surface of antigen presenting cells (APC). Binding of the processed peptides to the human leukocyte antigen (HLA) class II molecules HLA-DR, -DP or -DQ occurs in endosomal compartments and is facilitated by accessory molecules. These HLA class II molecules are highly polymorphic within the population and for many immunogenic peptides degenerate binding to different MHC-class II molecules has been described. This feature is of interest for instance in vaccine development as it would allow presentation of such T cell epitopes on APC within a wide population range [2,3]. Besides, also for TCR a plasticity in ligand recognition, i.e. degeneracy in peptide reactivity as well as cross-recognition in the context of distinct MHC-molecules has been found [4,5].

In Europe and parts of Asia, mugwort (Artemisia vulgaris) pollen causes the most prevalent pollen allergy in late summer and autumn. Mugwort pollen contains only one major allergen, Art v 1, recognized by IgE in 95\% of mugwort pollen-allergic individuals [6]. In contrast to other relevant major allergens, Art v 1 contains only one single immunodominant $\mathrm{T}$ cell epitope, Art $\mathrm{v}$ 125-36 (KCIEWEKAQHGA) which is recognized by more than $80 \%$ of Art v 1-reactive mugwort pollen-allergic patients [7]. The presentation of Art $\mathrm{v} 1_{25-36}$ is exclusively HLADR-restricted and is highly associated with the HLADR1 phenotype [8]. Other associations between HLA class II phenotypes and allergic responses to major allergens are by far less strong [9].

Although the $\mathrm{T}$ cell response to Art $\mathrm{v} 1_{25-36}$ is clearly associated with HLA-DR1, we previously found Art $\mathrm{v}$ $1_{25-36}$-specific $\mathrm{T}$ cells restricted by HLA-DR3, -DR16 or -DR15 in the minor fraction of DR1-negative patients [10]. Accordingly, the peptide showed degenerate high affinity binding to several HLA-DR alleles [10]. We also observed that specific $\mathrm{T}$ cell activation was inducible by APC expressing DR4 or other non-DR1-HLA alleles suggesting TCR cross-recognition. Although DR4 seemed to be able to present the immunodominant Art v 1 epitope, the frequency of DR4 in our Art v 1-reactive patient group $(10,7 \% ; \mathrm{n}=75)$ is not increased compared to the normal population $(16 \% ; n=100)$. Notably, we have also not been able to isolate DR4-restricted Art v $1_{25-36}$-specific $\mathrm{T}$ cells from DR1-negative, DR4-positive subjects $(n=8 / 75)$ during our intense attempts to establish TCL and TCC from mugwort-allergic patients. This discrepancy between HLA-DR4 and -DR1 is striking, as both molecules belong to the same HLA-DR supertype that includes DRB1*01:01, DRB5*01:01, DRB1*15:01, DRB1*04:01, DRB1*07:01, DRB1*09:01, and DRB1*13:02 which all show overlapping peptide-binding repertoires [11]. It is unlikely that the uniform $\mathrm{T}$ cell response to Art $\mathrm{v} 1$ is due to a restricted TCR V $\beta$ family repertoire, as we found a broad spectrum of $V \beta$ families in Art $v$ $1_{25-36}-$ specific cells even within single patients [8]. For DR4-restriction, in general also a broad TCRV $\beta$ usage has been reported $[12,13]$ arguing against a lack of specificity for Art v $1_{25-36}$ in the naïve TCR repertoire. Thus, currently there is no explanation why the DR1phenotype is highly prevalent in patients who recognize Art v $1_{25-36}$.

In this study, we investigated the molecular background leading to the preference of HLA-DR1 in the allergic response to the single immunodominant peptide of Art v 1. Potential differences between HLA-DR1 and -DR4 molecules regarding peptide binding and requirements for TCR recognition were addressed. We compared i) presentation of Art $\mathrm{v} 1_{25-36}$ by APC expressing either HLA-DR1 or -DR4 to allergen-specific T cell lines (TCL) and clones (TCC) and ii) in vitro binding of Art v $1_{25-36}$ to HLA-DR 1 and -DR4 molecules using mutated peptides; furthermore, iii) we performed computer simulations of Art v $1_{25-36}$-binding to both HLA-molecules using Molecular Dynamics (MD) simulations.

We found that the majority of Art v 1-specific $\mathrm{T}$ cells recognize synthetic Art $v 1_{25-36}$ and variants thereof as well as naturally processed peptides presented by DR1 and DR4 in a similar manner. Differences in minimal epitope recognition suggested structural differences for binding to the MHC peptide binding groove. MD simulation supported this conclusion demonstrating that the peptide is much more flexible at both ends and shows a detached structure at the C-terminus, whereas in DR4 the peptide keeps its extended conformation. Together, the strong bias of HLA-DR1 in the response to the immunodominant epitope of Art v 1 apparently is not primarily due to $\mathrm{MHC}$ /peptide binding per se, but rather seems to be based on structural differences in the TCRbinding site of the peptide/MHC (pMHC) complex.

\section{Methods}

\section{Patients}

Eleven mugwort pollen-allergic individuals with typical clinical history, i.e. recurrent rhinitis/conjunctivitis during late summer, positive skin prick test $(\geq 3 \mathrm{~mm})$ to mugwort pollen extract (ALK-Abello, 
Hørsholm, Denmark) and allergen-specific IgE (CAPFEIA $\geq 3$ ) to mugwort pollen (w6; Phadia, Uppsala, Sweden) were included. All subjects were sensitised to Art $\mathrm{v} 1$ as determined by IgE-immunoblots as described [7]. They expressed the following HLA-DR alleles: Pat.1 DRB1*01, DRB1*11; Pat. 2 DRB1*01, DRB1*15; Pat. 3 DRB1*01, DRB1*13; Pat. 4 DRB1*01, DRB1*03 ; Pat. 5 DRB1*01, DRB1*11; Pat. 6 DRB1*01, DRB1*11; Pat. 7 DRB1*1, DRB1*16; Pat. 8 DRB1*01, DRB1*13; Pat. 9 DRB1*01, DRB1*16, Pat. 10 DRB1*01, DRB1*03; Pat. 11 DRB1*01, DRB1*01; The study was approved by the ethics committee of the Medical University of Vienna (EK No. 497/2005) and informed consent was obtained from all individuals.

\section{Allergen and peptides}

Natural Art v 1 [6] was kindly provided by Matthias Egger (University of Salzburg, Austria). Synthetic peptides were obtained from Thermo Electronics (Ulm, Germany). Purity was $>90 \%$ as confirmed by HPLC.

\section{Peptide/MHC-binding assay}

HLA-DRB1*01:01 and -DRB1*04:01 molecules were purified from homozygous EBV cells by affinity chromatography using mAb L243 as described before [14]. Peptide binding to HLA-DR molecules was assessed by competitive ELISA. Dilutions were prepared in $10 \mathrm{mM}$ phosphate, $150 \mathrm{mM} \mathrm{NaCl}, \mathrm{mM}$ n-dodecyl $\beta$-D-maltoside, $10 \mathrm{mM}$ citrate $(\mathrm{pH}=6.0)$. The biotinylated reporter peptide $\left(\mathrm{HA}_{306-318}\right)$ was incubated with HLA-DRB1*01:01 or -DRB1*04:01molecules in the presence of serial dilutions of Art v $1_{25-36}$ or single mutant peptides thereof. Unlabelled $\mathrm{HA}_{306-318}$ peptide was also introduced to assess the validity of each experiment. After 24 hours of incubation at $37^{\circ} \mathrm{C}$, peptide/MHC-class II complexes were added to 96 well plates coated with L243 antibody and incubated at RT for 2 hours. Bound biotinylated peptides were detected by Streptavidin-alkaline phosphatase conjugate (GE healthcare, Saclay, France) and 4-methyl-umbelliferyl phosphate (Sigma-Aldrich, St. Quentin Fallavier, France) as substrate. Emitted fluorescence was measured at $450 \mathrm{~nm}$ upon excitation at $365 \mathrm{~nm}$ by a Gemini fluorometer (Molecular probes, St Grégoire, France). Data are expressed as peptide concentrations that prevented binding of $50 \%$ of the labelled reference peptide (IC50) $\mathrm{HA}_{306-318}$ (PKYVKQNTLKLAT). Two independent experiments were performed to evaluate competitive binding. Data were reported as relative affinity, i.e. ratio of the IC50 of the peptides to IC50 of the peptide $\mathrm{HA}_{306-318}$, to account for the differences of sensitivity of the binding assays.

\section{Allergen-specific $T$ cell lines (TCL) and T cell clones (TCC)} Art v $1_{25-36}$-specific TCL were established from peripheral blood mononuclear cells (PBMC) by allergen- specific stimulation with either mugwort pollen extract or recombinant Art v 1 (Biomay, Vienna, Austria) as previously described [7]. Art v $1_{25-36}$-specific TCC were established from these TCL by limiting dilution [7]. The two EBV-transformed B cell lines used as APC expressed comparable amounts of HLA-DR molecules (Figure 1A) as well as HLA-DQ, -DP- and the co-stimulatory molecules CD80, CD86, CD40 (not shown) on their surface. TCL or TCC $\left(5 \times 10^{4}\right.$ cells $)$ were stimulated for 3 days with irradiated (60 Gy) APC and peptides at an optimum concentration of $3 \mu \mathrm{M}$, if not indicated otherwise. Proliferation was assessed by ${ }^{3} \mathrm{H}$-thymidine uptake within the last 16 hours of incubation. Results are shown in delta $\mathrm{cpm}(\mathrm{dpm}=$ mean $\mathrm{cpm}$ of $\mathrm{T}$ cell proliferation with peptide - mean of $\mathrm{T}$ cell proliferation without peptide).

\section{Flow cytometry}

EBV-transformed B cell lines were stained with FITClabelled HLA-DR (L243), DP-(B7/21) and DQ-(SK10) antibodies, CD80 PE, CD86 PE (all BD Biosciences, Franklin Lakes, NJ) and anti CD40 FITC (BioLegend, San Diego, CA) and were analyzed on a FACSCanto with DIVA-software.

\section{HLA-typing}

Typing of HLA-DRB1-alleles was performed using a commercial SSO typing kit (DynalRELI SSO HLA-DRB Typing kit, Dynal, Bromborrough, UK). Samples with only a single detectable DRB1-allele were additionally typed by SSP (Dynal All set SSP DR low resolution, Dynal). High resolution typing was performed by nucleotide sequencing (BigDye Terminator Cycle Sequencing Kit, ABI, Foster City, CA).

\section{Computer simulation}

As starting structure we employed the x-ray structure of HIV GAG(p24)/HLA-DRB1*01:01, Protein Data Bank (PDB) [15] accession code [PDB:1sjh] [16], as it comprises HLA-DR1 in complex with a 13-mer peptide (PEVIPMFSALSEG) which also contains isoleucine as anchor residue similar to Art $\mathrm{v} 1_{25-36}$ (KCIEWEKAQHGA). We have previously shown that it is sufficient to mutate the side chains of the peptide and leave its backbone untouched to obtain reliable starting structures [17]. We selected the side-chain substitution tool SCWRL [18] to model Art v $1_{25-36}$ into the MHC binding groove, since we had found that this tool is the most accurate regarding peptide/MHC interactions $[17,19]$.

As the x-ray structure of HLA-DRB1*04:01 has not been resolved yet and HLA-DRB1*01:01 and HLADRB1*04:01 show a sequence identity of $91.58 \%$, we modelled the structure of DRB1*04:01 directly on the basis of the previously created HLA-DRB1*01:01 

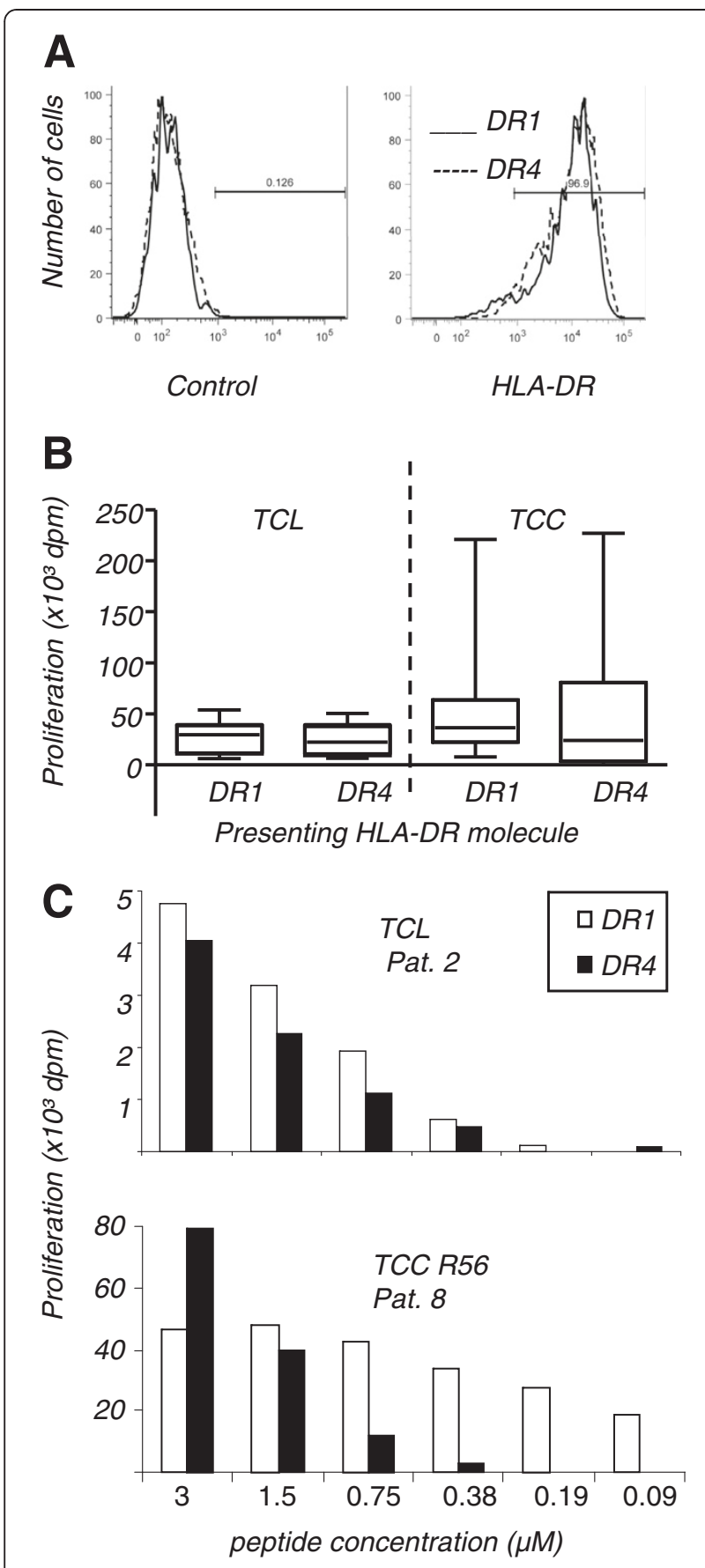

Figure 1 Presentation of Art $v \mathbf{1}_{25-36}$ by APC expressing HLADR1 or -DR4. A. HLA-DR expression on the EBV cell lines used as APC. B. Stimulation of TCL $(n=9)$ and TCC $(n=17)$ derived from 5 different subjects with an optimum concentration of Art $v 1_{25-36}$ peptide $(3 \mu \mathrm{M})$. Box plots indicating the median and quartile ranges are shown. Background proliferations for DR1 or DR4 plus T cells ranged from 1,002-27,612 cpm and 2406-21054 cpm for TCL and from 1525$4264 \mathrm{cpm}$ and 370-4270 cpm for TCC). C. T cell reactivity to different concentrations of Art $\vee 1_{25-36}$ presented by HLA-DR1 or -DR4 T cells were stimulated for 3 days with DR1- or DR4-expressing APC and proliferation was measured by ${ }^{3} \mathrm{H}$-thymidine uptake during the last 16 hours. Background proliferations were 2796/3056 cpm for Pat 2, 2247/1318 cpm for Pat 8. structure. The differing residues were mutated using SCWRL yielding 2 (pMHC) complexes with identical backbone structure and a few side chain mutations in the MHC.

To perform MD simulations we used the software package GROMACS 4 [20]. First, we immersed both complexes into distinct explicit artificial water boxes. Then we employed a steepest descent energy minimisation of the complexes to resolve spatial clashes and simulated warming to $310 \mathrm{~K}$ using position restraints. To provide insight into the dynamics of the pMHC complex at atomic scale, we subsequently carried out MD simulations [21] to investigate spatial rearrangements of molecular structures in water for a real time of $30 \mathrm{~ns}$. All further parameters of the simulation were set according to Omasits et al. [22].

We evaluated the resulting trajectories using the root mean square fluctuation (RSMF) which is defined as:

$$
\operatorname{RMSF}_{i}=\sqrt{\frac{1}{M} \sum_{k=1}^{M}\left(r_{i}\left(t_{k}\right)-\tilde{r}_{i}\right)^{2}}
$$

$\mathrm{M}$ is the number of frames, $r_{i}\left(t_{k}\right)$ is particle with number $i$ of complex $r$ at time $k$ and $\tilde{r}$ is the reference structure. This method essentially assesses flexibility of molecules and is implemented in GROMACS in the $g$ rms $f$ function. Furthermore, we calculated the solvent accessible surface area (SASA) [23] of the side chains of the peptide by rolling a sphere over them [24]. This method is implemented in GROMACS in the g sas function. Visual pre-screening was performed using Visual Molecular Dynamics software (VMD, NIH, University of Illinois) [25] and the plug-in vmdICE [26].

\section{Statistics}

Significance levels were calculated by using Wilcoxon signed rank test (SPSS 10.0, Chicago, IL).

\section{Results}

Presentation of Art v $1_{25-36}$ and mutant peptides by HLADR1 and -DR4

To compare the peptide presenting capacity of HLADR1 and DR4, 9 Art v 125-36-specific TCL from 7 different patients and 17 Art $\mathrm{v} 1_{25-36}$-specific TCC derived from 5 different patients were stimulated with the synthetic Art $v 1_{25-36}$ 12-mer peptide in the presence of DR1- or DR4-expressing APC. At optimum peptide concentrations, TCL proliferated slightly less with DR4, although no statistically significant difference was found (Figure 1B; median: 31,630 dpm and 25,985 dpm; $\mathrm{p}=0.314)$. A similar picture was found with the vast majority of TCC with median proliferations of $36,640 \mathrm{dpm}$ to DR1 and $24,125 \mathrm{dpm}$ to DR4 ( $\mathrm{p}=0.193$; Figure $1 \mathrm{~B})$. 
3/17 (18\%) TCC did not respond to presentation by DR4. At lower concentrations of Art $\mathrm{v} 1_{25-36}$, APC expressing DR1 outperformed those expressing DR4 (Figure 1C).

Next we determined the minimal $\mathrm{T}$ cell epitopes within Art v $1_{25-36}$ by employing truncated peptides for stimulation of Art $v 1_{25-36}$-reactive TCL ( $n=7 ; 6$ different donors) and one TCC (Figure 2A). Minimal epitopes ranged from 5 to 9 aa for DR1 and from 8 to11 aa for DR4. Within these minimal epitopes, the central indispensable aa common in all experiments were EWEKA for DR1 and IEWEKAQH for DR4. In 1 TCC (Pat. 8) and 3 TCL (Pat. 3, 4, 7), the minimal epitope was shorter for DR1 than for DR4 (Figure 2A). For 3/7 Art v $1_{25-36^{-}}$ specific TCL we found identical minimal epitopes for presentation by either DR-molecule.

In general, for peptides which bind to DR1 or DR4, the aa in positions $\mathrm{p} 1$ (defined as residue which provides the first anchor), p4, p6 and p9 are supposed to be relevant for HLA-binding, interacting with pockets on the bottom of the binding groove, so that the surfaceexposed intermittent aa residues at p2/3, p5, p7/8 can interact with the TCR $[11,27]$. I27 has previously been identified as anchor residue p1 of Art $\mathrm{v} 1_{25-36}$ [8]. To identify critical aa residues of Art $\mathrm{v} 1_{25-36}$ when presented either by DR1 or DR4, a set of peptide analogues with single aa substitutions was used for T cell stimulations. Besides the crucial anchor residue I27 (p1), W29 (p3) turned out to be indispensable for $\mathrm{T}$ cell reactivity in response to presentation by DR1 and DR4 in all TCL (from 6 different donors) and TCC (from 2 donors) tested (Figure 2B). Moreover, E28 (p2), E30 (p4) and K31 (p5) were critical for more than 70\% of the T cell responses. The residues C26 (p-1), Q33 (p7), H34 (p8) and A36 (p10) turned out to be additionally important for $30-50 \%$ of $\mathrm{T}$ cells, again irrespective of presentation by DR1 or DR4 (Figure 2B). A32 (p6) and G35 (p9) represented dispensable residues, possibly due to their small side chains which limit molecular interactions.

Taken together, for the $\mathrm{T}$ cell recognition of Art $\mathrm{v} 1_{25-36}$ we found indications for a shorter minimal epitope in DR1, but no marked differences in the position and number of residues crucial for interaction with the two HLAmolecules.

\section{Presentation of naturally-processed Art $\mathbf{v}$ 1-peptides by HLA-DR1 and -DR4 molecules}

The experiments performed so far had been based on synthetic 12-mer peptides which might bind directly to HLA-DR-molecules expressed on the surface of APC. To compare intracellular loading of processed Art v 1peptides onto DR1- and DR4-molecules, HLA-DR1- or

\begin{tabular}{|c|c|c|c|c|c|c|c|c|c|c|c|c|c|c|c|c|c|c|}
\hline \multirow[b]{2}{*}{ Truncated Peptides } & \multicolumn{12}{|c|}{$T C L$} & & & \multicolumn{2}{|c|}{ TCC } & & \\
\hline & Pat. 1 & & Pat. 2 & & Pat. & & Pat. 4 & & Pat. & & Pat.5 & & Pat. 6 & & $R 9 F$ & at. 7 & & \\
\hline Art v $125-36$ & $D R 1$ & $\overline{D R 4}$ & $D R 1$ & $D R 4$ & $D R 1$ & $D R 4$ & $D R 1$ & $D R 4$ & $D R 1$ & $D R 4$ & $D R 1$ & $D R 4$ & $D R 1$ & $D R 4$ & $D R 1$ & $D R 4$ & & \\
\hline KCIEWEKAQHGA & + & + & + & + & + & + & + & + & + & + & + & + & + & + & + & + & & \\
\hline CIEWEKAQHGA & + & + & + & + & + & $(+)$ & + & - & + & + & + & + & + & + & + & + & K & \\
\hline IEWEKAQHGA & $(+)$ & - & + & + & + & - & - & - & - & - & + & + & + & + & + & + & C & \\
\hline EWEKAQHGA & - & - & - & - & - & - & - & - & - & - & - & - & + & - & + & - & 1 & p1 \\
\hline WEKAQHGA & - & - & - & - & - & - & - & - & - & - & - & - & $(+)$ & - & - & - & E & $p 2$ \\
\hline$E K A Q H G A$ & - & - & - & - & - & - & - & - & - & - & - & - & - & - & - & - & w & p3 \\
\hline$K A Q H G A$ & - & - & - & - & - & - & - & - & - & - & - & - & - & - & - & - & E & p4 \\
\hline KCIEWE & - & - & - & + & - & - & - & - & - & - & - & - & - & - & - & $(+)$ & $\mathrm{K}$ & p5 \\
\hline KCIEWEK & - & - & - & - & - & - & - & - & - & - & - & - & - & - & - & - & A & $p 6$ \\
\hline KCIEWEKA & - & - & - & - & - & - & - & - & - & - & - & - & + & - & + & - & $Q$ & $p 7$ \\
\hline KCIEWEKAQ & - & - & - & - & - & - & - & - & - & - & - & - & + & - & + & - & $\mathrm{H}$ & $p 8$ \\
\hline KCIEWEKAQH & 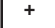 & + & 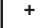 & + & - & - & + & + & + & + & + & + & 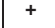 & + & + & + & $G$ & p9 \\
\hline KCIEWEKAQHG & + & + & + & + & + & + & + & - & + & + & + & + & + & + & + & + & & \\
\hline
\end{tabular}

\begin{tabular}{|c|c|c|c|c|c|c|c|c|c|c|c|c|c|c|c|c|c|c|c|c|c|}
\hline & \multicolumn{12}{|c|}{$T C L$} & \multicolumn{9}{|c|}{$T C C$} \\
\hline Mutant Peptides & Pat. 1 & & Pat. 2 & & Pat. & & Pat. & & Pat. 7 & & Pat. 8 & & $R 97 P$ & at. 7 & $R 114 \mathrm{~F}$ & at. 7 & $R 24 P$ & at. 7 & $R 51 P_{a}$ & at. 9 & \\
\hline Art v 1 25-36 & $D R 1$ & $D R 4$ & $D R 1$ & $D R 4$ & $D R 1$ & $D R 4$ & $D R 1$ & $D R 4$ & $D R 1$ & $D R 4$ & $D R 1$ & $D R 4$ & $D R 1$ & $D R 4$ & $D R 1$ & $D R 4$ & $D R 1$ & $D R 4$ & $D R 1$ & $D R 4$ & \\
\hline KCIEWEKAQHGA & + & + & + & + & + & + & + & + & + & - & + & + & + & + & + & + & + & + & + & - & \\
\hline ACIEWEKAQHGA & + & + & + & + & + & + & + & + & + & + & + & + & nd & nd & + & + & nd & nd & + & + & K \\
\hline KAIEWEKAQHGA & + & - & + & + & + & + & - & + & - & + & + & + & - & - & + & + & - & - & + & + & o \\
\hline KCAEWEKAQHGA & - & - & + & - & - & - & + & - & - & - & - & - & - & - & - & - & - & - & - & - & - \\
\hline KCIAWEKAQHGA & - & - & - & - & + & + & - & - & - & - & - & - & - & - & + & + & - & - & - & - & JE \\
\hline KCIEAEKAQHGA & - & - & - & - & - & - & - & - & - & - & - & - & - & - & - & - & - & - & - & - & IV \\
\hline KCIEWA & - & - & - & - & + & + & - & - & - & + & - & - & + & + & - & - & - & - & - & - & E \\
\hline KCIEWEAAQHGA & - & - & + & - & - & - & - & - & - & - & - & - & - & - & - & - & + & + & - & - & $\mathrm{K}$ \\
\hline KCIEWEKGQHGA & + & + & + & + & + & - & + & + & + & - & + & + & + & + & + & + & + & - & + & + & A \\
\hline KCIEWEKAAHGA & - & + & - & - & - & - & + & + & + & $(+)$ & - & - & + & + & - & + & + & - & + & + & 0 \\
\hline KCIEWEKAQAGA & + & + & + & + & - & - & + & - & - & + & - & - & + & + & - & - & + & + & + & - & H \\
\hline KCIEWEKAQHAA & + & + & + & + & + & + & + & + & - & + & + & + & + & + & + & + & + & + & - & - & $\mathrm{G}$ \\
\hline KCIEWEKAQHGG & + & + & + & + & + & - & + & + & - & + & + & + & + & - & + & + & nd & nd & - & - & \\
\hline
\end{tabular}

No. of experiments tolerating mutations

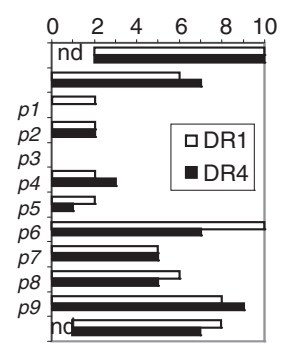

Figure 2 Mapping of minimal T cell epitopes and critical amino acids within Art v $\mathbf{1}_{25-36}$ presented by HLA-DR1 or -DR4. T cell reactivity was tested with truncated and single mutant Art $\vee 1_{25-36}$-peptides. T cells were stimulated for 3 days with DR1- or DR4-expressing APC and proliferation was measured by ${ }^{3} \mathrm{H}$-thymidine uptake during the last 16 hours; $(+)$ denotes $>50 \%$ and $(-)>70 \%$ reduction of proliferation induced by KCIEWEKAQHGA (dpm). Background proliferations for DR1 or DR4 plus T cells ranged from 1,002-27,612 cpm and 2406-21054 cpm for TCL and from 1525-4264 cpm and 370-4270 cpm for TCC. 
DR4-expressing APC were incubated with titrated amounts of Art v 1 and used to stimulate Art v $1_{25-36^{-}}$ specific $\mathrm{T}$ cell cultures. As observed before with synthetic peptides, the Art v $1_{25-36}$-specific TCL and 2 TCC proliferated equally well to DR1 or DR4 at optimum concentrations. However, at lower concentrations DR1 tended to be more effective for T cell stimulation (Figure 3).

\section{Binding of Art v $\mathbf{1}_{\mathbf{2 5 - 3 6}}$ to HLA-DR1 and -DR4 molecules} The binding capacity of Art $\mathrm{v} 1_{25-36}$ to HLA-DRB1*01:01 and $-D R B 1 * 04: 01$ was assessed in competition studies using $\mathrm{HA}_{306-318}$ as reporter peptide (Table 1). $\mathrm{HA}_{306-318}$, a high affinity binder to both DR1 and DR4, inhibited itself at an IC50 of 1.8 and $11 \mathrm{nM}$, respectively. Art v $1_{25-36}$ was found to bind only 15 -fold less to both HLA molecules and hence can still be considered a strong binder for both HLA-molecules. For comparison, we used the neighbouring 12-mer peptides Art v $1_{22-33}$ and Art v 1 19-30

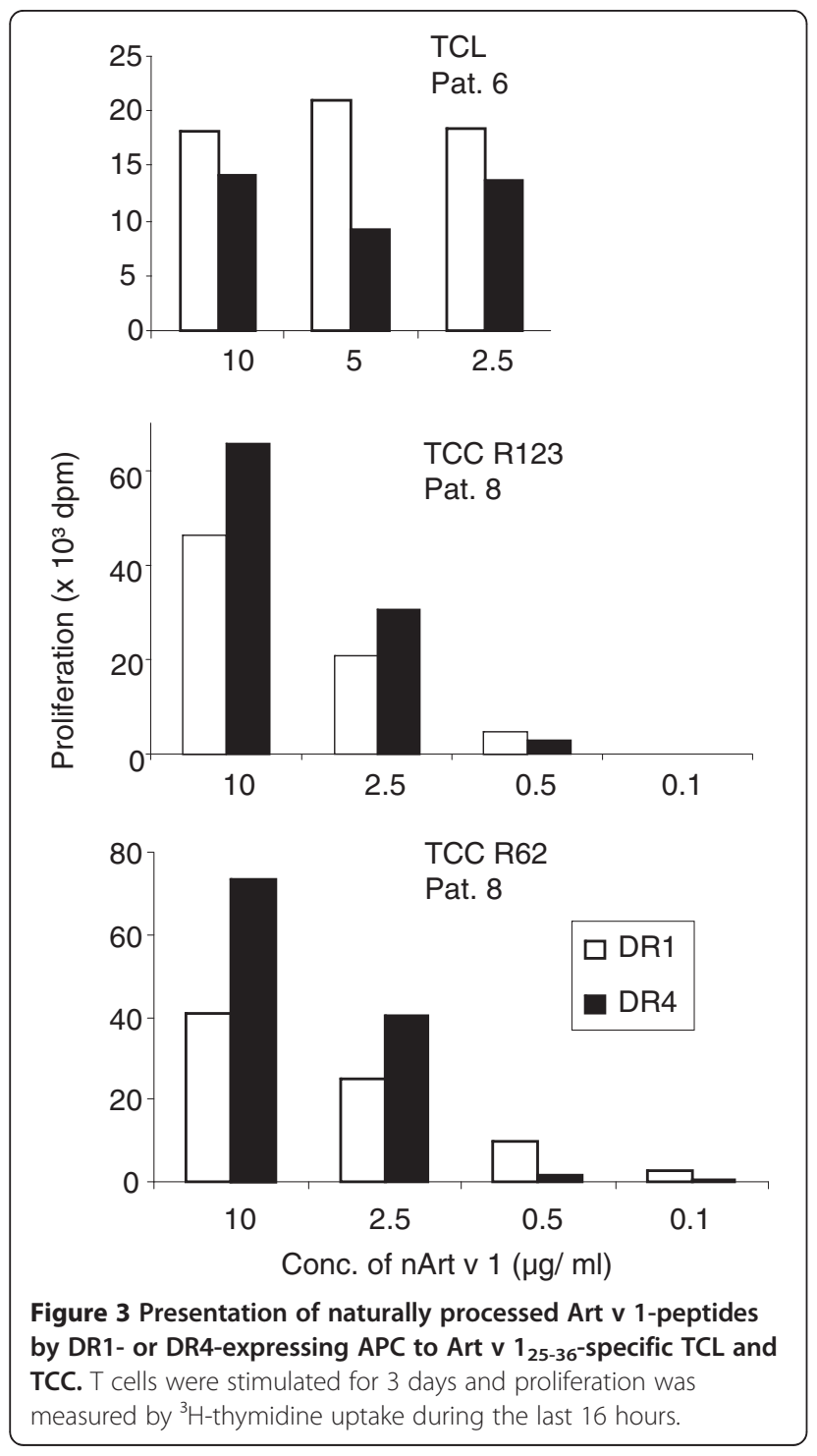

which showed weak and negligible binding to DR1 or DR4 molecules, respectively.

To allocate the aa residues crucial for anchoring the peptide within the groove of the HLA molecule, peptides with single alanine/glycine substitutions at each amino acid position of Art v $1_{25-36}$ were used. For both HLAmolecules a marked loss of binding was obtained by replacing I27 (Table 1), clearly confirming that this is the anchor residue for HLA-DR1 [8] and indicating that the peptide uses the same anchor residue for binding to DR4. Weaker binding losses for either molecule were induced by glycine substitutions of original alanine residues (A32, A36), which might result from enhancement of the peptide flexibility due to the special properties of glycine. Substitution of W29 resulted in a slight effect on binding to DR1, but not to DR4.

\section{Computer simulation of Art $v \mathbf{1}_{25-36}$ bound to HLA-DR1 and -DR4}

In parallel to our in vitro experiments, we investigated structural differences at the level of $\mathrm{MHC} /$ peptide binding in silico by performing $\mathrm{MD}$ simulations. HLADRB1*01:01 and HLA-DRB1*04:01 show an aa sequence identity of $91.58 \%$. On this basis the overall tertiary structure is expected to be very similar (see Methods). However, the binding dynamics of Art $v 1_{25-36}$ to each HLA-molecule differed markedly during the simulation time of 30 ns (Figure 4A,B). The interaction with HLA-DR1 was characterized by highly flexible aa residues at the N- (K25) and C-terminus (H34, G35 and A38; Figure $4 \mathrm{C}$ ) of the peptide with the latter detaching from the binding groove (Figure 4A). In contrast, only A36 showed high flexibility in the interaction with HLA-DR4 (Figure 4C). The different dynamics of Art $\mathrm{v} 1_{25-36}$ binding to HLA-DRB1*01:01 and HLADRB1*04:01 were also reflected in the mean solvent accessible surface area (SASA) (Figure 4D). K31 and H34 show an increased SASA in HLA-DRB1*01:01 compared to DR4, while the SASA was decreased in the case of K25, E28, E30 and Q33. Interestingly, the increased flexibility of Art $\mathrm{v} 1_{25-36}$ in association with HLA-DRB1*01:01 was restricted solely to the peptide itself while the helices of the alpha- and beta-chains of DR1 and DR4 showed a similar behaviour in both molecules (Figure 4E,F). For I27 we found a low flexibility and an almost identical SASA in both complexes indicating that this residue is stably bound to the MHC in both cases.

\section{Discussion}

In spite of the strong association of the allergic immune response to Art v 1 with HLA-DR1, we have previously shown that the in vitro binding of its single major $\mathrm{T}$ cell epitope Art v $1_{25-36}$ to HLA-class II molecules and its recognition by $\mathrm{CD} 4+\mathrm{T}$ cells is degenerate $[7,8]$. In 
Table 1 Binding of Art v 1-peptides and mono-substituted peptides of Art v 125-36 to HLA-DRB1*01:01 and HLA-DRB 1*04:01

\begin{tabular}{|c|c|c|c|c|}
\hline \multirow[t]{2}{*}{ Peptide } & \multirow{2}{*}{$\begin{array}{l}\text { Amino Acid } \\
\text { sequence }\end{array}$} & \multicolumn{2}{|c|}{${ }^{\mathrm{a}}$ ratio of $\mathrm{IC}_{50}$ of Art v 1-peptides/HA ${ }_{306-318}$} & \\
\hline & & HLA-DR1 & HLA-DR4 & \\
\hline $\mathrm{HA}_{304-318}$ & PKYVKQNTLKLAT & 1 & 1 & \\
\hline Art $\vee_{119-30}$ & NKKCDKKCIEWE & $>55032$ & $>926$ & \\
\hline Art $\vee 1_{22-33}$ & CDKKCIEWEKAQ & 2016 & 756 & \\
\hline Art $\vee 1_{26-36}$ & KCIEWEKAQHGA & 14 & 15 & \\
\hline Ala-25 & ACIEWEKAQHGA & 9 & 21 & \\
\hline Ala-26 & KAIEWEKAGHGA & 16 & 3 & \\
\hline Ala-27 & KCAEWEKAQHGA & 2596 & $>926$ & p1 \\
\hline Ala-28 & KCIAWEKAQHGA & 6 & 11 & p2 \\
\hline Ala-29 & KCIEAEKAQHGA & 69 & 14 & p3 \\
\hline Ala-30 & KCIEWAKAQHGA & 6 & 28 & p4 \\
\hline Ala-31 & KCIEWEAAQHGA & 17 & 17 & p5 \\
\hline Gly-32 & KCIEWEKGQHGA & 63 & 149 & p6 \\
\hline Ala-33 & KCIEWEKAAHGA & 11 & 31 & p7 \\
\hline Ala-34 & KCIEWEKAQAGA & 8 & 17 & p8 \\
\hline Ala-35 & KCIEWEKAQHAA & 5 & 5 & p9 \\
\hline Gly-36 & KCIEWEKAQHGG & 74 & 242 & \\
\hline
\end{tabular}

${ }^{a}$ Relative binding activity. Binding of peptides to HLA-DR1 and -DR4 molecules was assessed by competitive inhibition of the high affinity interaction of biotinylated-HA $\mathrm{A}_{306-318}$ with HLA-DR1 and -DR4 as described in Methods. IC50 values were converted into relative activities (ratio between IC50 value and IC50 of $\mathrm{HA}_{306-318}$ ) to take into account the differences of sensitivity of the binding assays. The IC50 of HA $306-318$ was $1.8 \mathrm{nM}$ for HLA-DR1 and $11 \mathrm{nM}$ for -DR4.

addition to the vast majority of Art $\mathrm{v} 1_{25-36}$-specific, DR1-restricted TCC, a few TCC from DR1-negative patients have been isolated that were restricted by DR15, DR16 or DR3. Although DR4 in general has similar peptide binding characteristics [11] as DR1 and binds Art v $1_{25-36}$ which can be cross-recognized by DR1-restricted TCC in vitro [10], no Art v 125-36-specific DR4-restricted TCC have been isolated so far. Therefore, we expected differences in Art v $1_{25-36}$-peptide binding and $\mathrm{T}$ cell activation between HLA-DR1 and -DR4 and compared anchor residues and minimal epitopes of the peptide to elucidate the molecular background for the DR1-biased T cell response to Art v $1_{25-36}$ in more detail. To find further support for structural differences between Art v $1_{25-36}$ bound to HLA-DR1 or HLA-DR4 we also applied MD simulations to the respective pMHC complexes. As there was no crystal structure available for HLA-DR4, our computer simulations on peptide/HLA-DR4 interactions were performed on a modeled structure based on the $\mathrm{x}$-ray structure of HLA-DR1. Nevertheless, this model of HLADRB1*04:01 can be assumed to be reliable, as the sequence identity between DR1 and DR4 is above $90 \%$ and models based on a template with a sequence identity $>50 \%$ are generally regarded as highly accurate [28]. Furthermore, major changes in the fold of $\mathrm{MHC}$ molecules are extremely unlikely since the MHC superfamily shares a common overall shape [29].
First, we confirmed T cell cross-recognition of Art v $1_{25-36}$ presented by APC expressing DR4. All T cell cultures used in this study were derived from DR1-positive donors and had most probably been selected by this MHC class II restriction element. Still, the vast majority of TCC (82\%) recognized Art v $1_{25-36}$ presented by DR4 indicating degenerate TCR recognition (Figure 1B). However, at limiting peptide concentrations DR1expressing APC outperformed DR4-expressing APC in their stimulating capacity (Figure 1C). Natural processing of antigens generates a pool of peptides with different length (mostly 15-18 aa) [30,31] and their intracellular loading into MHC-class II molecules underlies editing by HLA-DM [32]. Similar to our synthetic, 12-mer Art v 1-peptides, the peptides resulting from natural processing were also loaded efficiently into both DR1- and DR4 molecules as detected by the re-

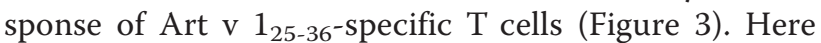
again, DR1-expressing APC performed better at lower peptide concentrations than DR4-expressing APC.

Next, differences in the binding behaviour of Art $\mathrm{v}$ $1_{25-36}$ to DR1- and DR4-molecules and T cell stimulatory capacity were addressed. In vitro binding assays with isolated HLA-molecules and single aa mutants of Art v $1_{25-36}$ confirmed peptide-binding to DR1-molecules with high affinity and residue I27 as p1-anchor [8]. Moreover, we found a similar binding affinity and again I27 as 


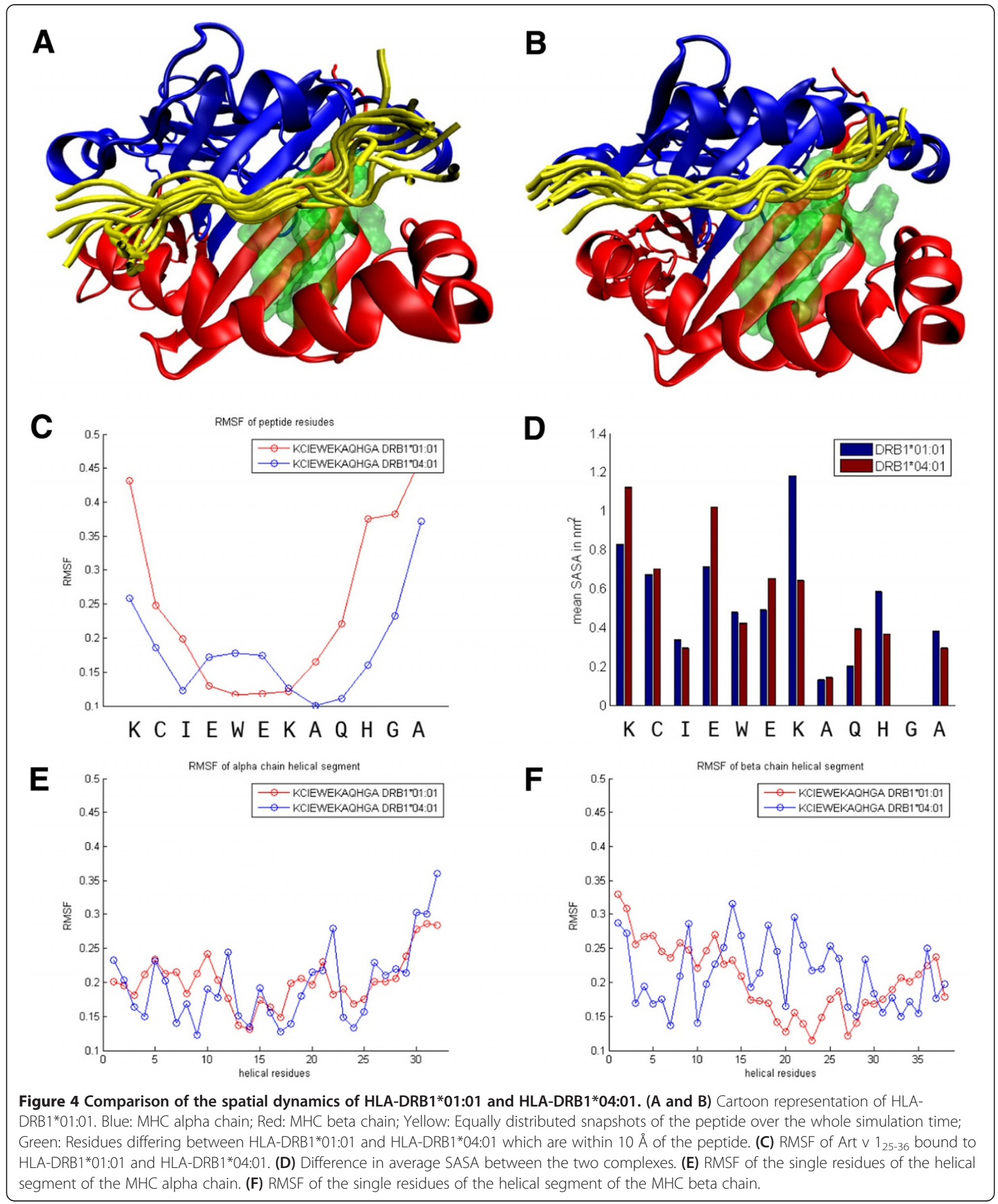

p1-anchor for binding to DR4-molecules (Table 1). The latter was consistent with our computer simulation data. Notably, the residues at p4, p6, or p9 (E30, A32, G35) which in general can bind to additional secondary binding pockets in the groove of DR1 and DR4, had no measurable further supporting effects for Art v $1_{25-36}$-binding to either DR molecule, again emphasizing the anchor role of I27. A and G by nature do not 
provide suitable side chains for anchoring and also proved not to be relevant for $\mathrm{T}$ cell activation (Figure $2 \mathrm{~B}$ ). On the other hand, E30 (p4) turned out to be important for most $\mathrm{T}$ cell responses (Figure 2). This finding might indicate that, instead of interacting with the HLA-molecule, E30 may interact with the TCR together with its neighbouring aa W29 (p3). However, the calculated surface exposition (SASA) of E30 (p4) obtained by computer modelling was relatively low (Figure 4F) and rather suggested interaction with the MHC molecule. In this case, the simultaneous presence of the all-dominant I27 in the A30-mutant peptide apparently prevented in vitro detection of secondary MHC-binding residues.

Overall, the position and number of Art $\mathrm{v} 1_{25-36^{-}}$ residues crucial for $\mathrm{T}$ cell reactivity was similar in response to DR1- or DR4-molecules and focussed on the 5 aa stretch from I27 to K31. The indispensible residues E28 (p2) W29 (p3), K31 (p5) are located in the core of the minimal epitopes found. Less frequently Q33 (p7) and H34 (p8) also formed part of the minimal epitopes (Figure 2A). Due to their increased flexibility and higher surface exposition of H34 in DR1 (Figure 4E,F), these aa may contribute to a more intense TCR-interaction. An influence of peptide flanking residues ( $\mathrm{p}-1$ and $\mathrm{p} 10$ ) on $\mathrm{T}$ cell responses was also observed in a few experiments, but was not restricted to either DR1- or DR4-presentation.

Although we found similar affinities and structures (i.e. the same register) for Art $\mathrm{v} 1_{25-36}$-binding to DR1 and DR4 (Table 1), for more than $50 \%$ of the T cells the minimal epitope was shorter for the DR1-bound peptide (Figure 2A). In silico, differences in peptide flexibility, indicated by RMSF, became obvious between Art v $1_{25-36}$ bound to HLA-DR1 or HLA-DR4 with advanced simulation time. In DR1 the peptide was more flexible at both ends and detached from the MHC-molecule at the $\mathrm{C}$ terminus (Figure $4 \mathrm{~A}-\mathrm{C}$ ). In essence, the parts of the Art $\mathrm{v}$ 1-peptide with lower ranges in RMSF, i. e. less flexibility, reflect the common core of the minimal peptide regions as defined by our in vitro experiments for DR1 (IEWEK) or DR4 (CIEWEKAQH). Thus these peptide ranges might define the parts of Art $v 1_{25-36}$ buried in the MHC-binding groove. Further indications for structural differences between the Art $v 1_{25-36} / \mathrm{DR} 1$ or -DR4 complex were the overall discrepancies obtained for surface exposition (SASA, Figure 4D). As compared to DR4, the C-terminal aa residues in DR1 were more solvent-exposed, while the $\mathrm{N}$-terminal residues were less exposed. In addition, K31 at position $\mathrm{p} 5$, classically the central solvent TCR-exposed residue in MHC class II [33], showed a markedly higher SASA value in complex with DR1 than with DR4. These differences may indicate better access of the TCR to the core and C-terminus of the Art v 1-peptide in DR1.

Our simulations were based on the crystal structure of the 13-mer peptide HIV Gag p24 ${ }_{34-46}$ PEVIPMFSALSEG bound to HLA-DRB1*0101 [PDB:1sjh]. Interestingly, a congruent crystal structure exists [PDB:1sje] representing HLA-DRB1*01:01 with the 15-mer peptide HIV Gag p24 34-48 containing 2 additional C-terminal aa residues $(\mathrm{A}, \mathrm{T})$. While the $13 \mathrm{~N}$-terminal residues of the peptide show an essentially identical spatial arrangement as the Art v 1-complex at the initial configuration [16,26], these two residues reach out of the $\mathrm{MHC}$ binding groove and build a hairpin turn which has been reported to be essential for T cell recognition of this HIV Gag p24-epitope [16,34]. Intriguingly, this hairpin structure resembles certain substates of the detached C-terminal end of the Art v $1_{25-36} /$ HLA-DRB1*01:01 complex as shown in Figure 5. Within 61 published crystal structures of pMHC class II molecules that contain at least short peptide flanking regions, we found in about $10 \%$ of them structures divergent from the canonical stretched conformation such as detachments, loop-like structures or hairpin turns. These structures most likely are important for T cell reactivity. We have previously reported effects of such protruding peptide segments on $\mathrm{T}$ cell activation for peptide flanking regions [34] and altered peptide ligands with high sequence similarity [35]. Thus, we suggest that the $\mathrm{C}$-terminal Art $\mathrm{v} 1_{25-36}$-residues protruding from the peptide binding groove are relevant for interactions with TCR in vivo.

An alternative interpretation for the lack of DR4responses to Art v 1 in the population may be a potential negative influence of flanking regions in naturally processed peptides selectively on HLA-DR4. However, the fact that DR1-restricted T cells also respond well to naturally processed, DR4-presented Art v 1 (Figure 3) at

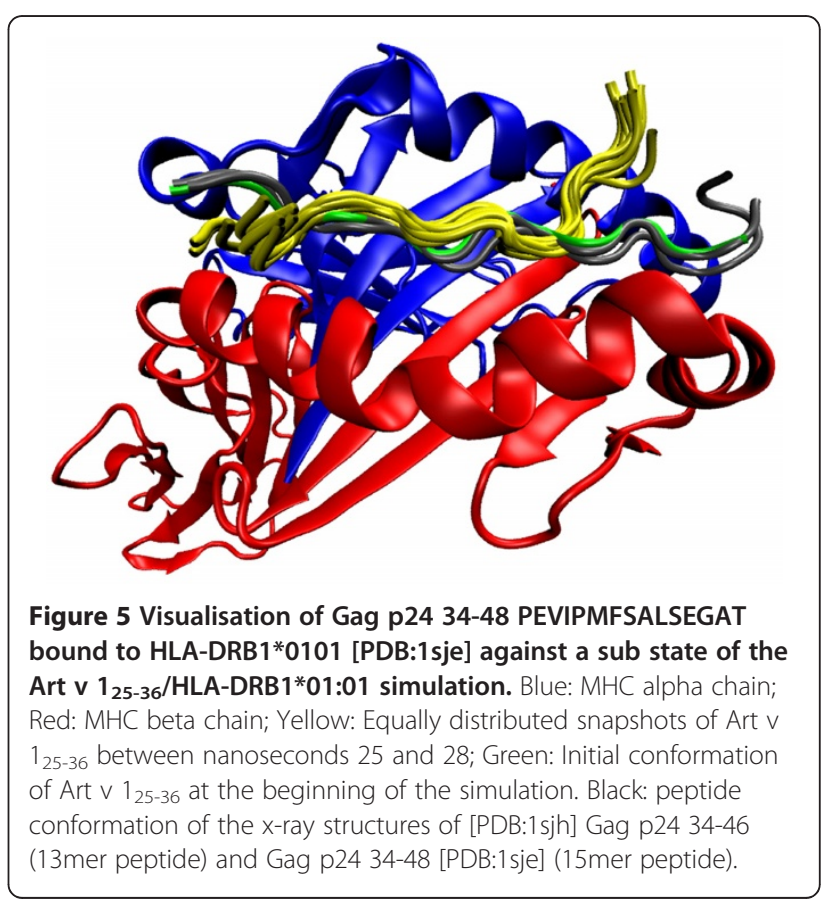


optimum concentrations indicates that these effects would only be of minor relevance.

In summary, compared to DR4, DR1 allowed T cell activation of shorter minimal epitopes within Art v $1_{25-36}$ and at lower peptide concentrations. However, the differences obtained in our experimental assays may not clearly explain the high association of Art $\mathrm{v} 1_{25-36^{-}}$ recognition and HLA-DRB1*01. We used allergenspecific effector $\mathrm{T}$ cells with a very high sensitivity for antigen-specific activation in our study. However, the threshold level for activation of naïve $\mathrm{T}$ cells is much higher. Therefore, we suggest that at much lower allergen-concentrations, i.e. at conditions present in the human body, the observed differences become much more relevant in vivo, so that DR1- but not DR4expressing APC are capable of activating cognate T cells. This assumption would be backed by the fact that we could not establish DR4-restricted TCC from any of our DR4-expressing mugwort-allergic patients.

\section{Conclusions}

We used the $\mathrm{T}$ cell response to the immunodominant epitope of Art $\mathrm{v} 1$ as a model to study $\mathrm{p} / \mathrm{MHC} / \mathrm{TCR}$ interactions in order to explain why a peptide with degenerate MHC-class II binding behaviour causes primarily HLA-DR1-restricted immune responses. The combination of experimental and in silico data suggested that TCR recognition of this peptide in the context of DR1 may be promoted by distinctive structural features in the pMHC complex leading e.g. to superior TCR accessibility and preferential priming in vivo.

\section{Abbreviations}

Aa: Amino acid; APC: Antigen presenting cell; HLA: Human leukocyte antigen; $\mathrm{IC}_{50}$ : Concentration causing half-maximum inhibition; MD: Molecular Dynamics; MHC: Major histocompatibility complex; pMHC: Peptide/major histocompatibility complex; PDB: Protein Data Bank; RMSF: Root mean square fluctuation; SASA: Solvent accessible surface area; TCR: T cell receptor; Th: T helper; TCL: T cell line/s; TCC: T cell clone/s.

\section{Competing interests}

$\mathrm{BB}$ is consultant for Biomay. All other authors declare that they have no competing interests.

\section{Authors' contributions}

BJS and BK conceived of the study; GF and BB participated in its design and interpretation; BK did the computer simulation studies; BJS and DVH carried out the T cell experiments; IF and GF performed HLA-typing and analysed the data; BM did the HLA-binding assays; WS contributed with materials and supervision; CE provided clinical expertise; BJS and BK prepared the manuscript; BB and GF helped to draft the manuscript. All authors read and approved the final manuscript.

\section{Acknowledgements}

We thank Matthias Egger for providing purified natural Art v 1. We are grateful to Claudia Kitzmüller for critically reading the manuscript. This study was supported by funds from the Austrian Science Fund: P20011-B13, SFBF4610 and P22258-B12, the Christian Doppler Society, the Oesterreichische Nationalbank (Anniversary Fund, project No.13860) and Biomay (Vienna, Austria).

\section{Author details}

'Department for Biomedical Computersimulation and Bioinformatics, Medical University of Vienna, Vienna, Austria. ${ }^{2}$ Department of Blood Group Serology, Medical University of Vienna, Vienna, Austria. ${ }^{3}$ Department of

Pathophysiology and Allergy Research, Medical University of Vienna, Vienna, Austria. ${ }^{4} \mathrm{CEA}$, iBiTecS, Service d'Ingénierie Moléculaire des Protéines (SIMOPRO), Gif-sur-Yvette, France. ${ }^{5}$ Allergieambulatorium Reumannplatz, Vienna, Austria. ${ }^{6}$ Christian-Doppler Laboratory for Immunomodulation, Medical University of Vienna, Vienna, Austria.

Received: 15 May 2012 Accepted: 2 August 2012

Published: 8 August 2012

\section{References}

1. Romagnani $\mathrm{S}$ : The role of lymphocytes in allergic disease. J Allergy Clin Immunol 2000, 105:399-408.

2. Hammer J, Valsasnini P, Tolba K, Bolin D, Higelin J, Takacs B, Sinigaglia F: Promiscuous and allele-specific anchors in HLA-DR-binding peptides. Cell 1993, 74:197-203.

3. Panina-Bordignon P, Tan A, Termijtelen A, Demotz S, Corradin G, Lanzavecchia A: Universally immunogenic T cell epitopes: promiscuous binding to human MHC class II and promiscuous recognition by T cells. Eur J Immunol 1989, 19:2237-2242.

4. Wilson DB, Wilson DH, Schroder K, Pinilla C, Blondelle S, Houghten RA, Garcia KC: Specificity and degeneracy of T cells. Mol Immunol 2004, 40:1047-1055

5. Ou D, Mitchell LA, Tingle AJ: HLA-DR restrictive supertypes dominate promiscuous $T$ cell recognition: association of multiple HLA-DR molecules with susceptibility to autoimmune diseases. J Rheumatol 1997, 24:253-261.

6. Himly M, Jahn-Schmid B, Dedic A, Kelemen P, Wopfner N, Altmann F, van Ree R, Briza P, Richter K, Ebner C, et al: Art v 1, the major allergen of mugwort pollen, is a modular glycoprotein with a defensin-like and a hydroxyproline-rich domain. Faseb J 2003, 17:106-108.

7. Jahn-Schmid B, Kelemen P, Himly M, Bohle B, Fischer G, Ferreira F, Ebner C The $T$ cell response to Art $v 1$, the major mugwort pollen allergen, is dominated by one epitope. J Immunol 2002, 169:6005-6011.

8. Jahn-Schmid B, Fischer GF, Bohle B, Fae I, Gadermaier G, Dedic A, Ferreira F, Ebner $C$ : Antigen presentation of the immunodominant T-cell epitope of the major mugwort pollen allergen, Art v 1 , is associated with the expression of HLA-DRB1 *01. J Allergy Clin Immunol 2005, 115:399-404.

9. Jahn-Schmid B, Pickl WF, Bohle B: Interaction of allergens, major histocompatibility complex molecules, and T cell receptors: a 'menage a trois' that opens new avenues for therapeutic intervention in type I allergy. Int Arch Allergy Immunol 2011, 156:27-42.

10. Jahn-Schmid B, Sirven P, Leb V, Pickl WF, Fischer GF, Gadermaier G, Egger M, Ebner C, Ferreira F, Maillere B, et al: Characterization of HLA class II/ peptide-TCR interactions of the immunodominant T cell epitope in Art v 1, the major mugwort pollen allergen. J Immunol 2008, 181:3636-3642.

11. Southwood S, Sidney J, Kondo A, del Guercio MF, Appella E, Hoffman S, Kubo RT, Chesnut RW, Grey HM, Sette A: Several common HLA-DR types share largely overlapping peptide binding repertoires. J Immunol 1998, 160:3363-3373.

12. Grunewald J, Shankar N, Wigzell $H$, Janson $\mathrm{CH}$ : An analysis of alpha/beta TCR $\checkmark$ gene expression in the human thymus. Int Immunol 1991, 3:699-702.

13. He X, Rosloniec EF, Myers LK, McColgan WL, 3rd Gumanovskaya M, Kang AH, Stuar JM: T cell receptors recognizing type II collagen in HLA-DRtransgenic mice characterized by highly restricted $\mathrm{V}$ beta usage. Arthritis Rheum 2004, 50:1996-2004.

14. Texier C, Pouvelle $\mathrm{S}$, Busson M, Herve M, Charron D, Menez A, Maillere B: HLA-DR restricted peptide candidates for bee venom immunotherapy. J Immunol 2000, 164:3177-3184.

15. Berman HM, Westbrook J, Feng Z, Gilliland G, Bhat TN, Weissig H, Shindyalov IN, Bourne PE: The Protein Data Bank. Nucleic Acids Res 2000, 28:235-242.

16. Zavala-Ruiz Z, Strug I, Walker BD, Norris PJ, Stern L: A hairpin turn in a class II MHC-bound peptide orients residues outside the binding groove for T cell recognition. Proc Natl Acad Sci U S A 2004, 101:13279-13284.

17. Knapp B, Omasits U, Schreiner W: Side chain substitution benchmark for peptide/MHC interaction. Protein Sci 2008, 17:977-982. 
18. Canutescu AA, Shelenkov AA, Dunbrack RL: Jr.: A graph-theory algorithm for rapid protein side-chain prediction. Protein Sci 2003, 12:2001-2014.

19. Knapp B, Omasits U, Frantal S, Schreiner W: A critical cross-validation of high throughput structural binding prediction methods for pMHC. J Comput Aided Mol Des 2009, 23:301-307.

20. Hess B, Kutzner C, VanderSpoel D, Lindahl E: GROMACS 4: Algorithms for Highly Efficient, Load-Balanced, and Scalable Molecular Simulation. J Chem Theory Comput 2008, 4:435-447.

21. Karplus M, McCammon JA: Molecular dynamics simulations of biomolecules. Nat Struct Biol 2002, 9:646-652.

22. Omasits U, Knapp B, Neumann M, Steinhauser O, Stockinger H, Kobler R, Schreiner W: Analysis of Key Parameters for Molecular Dynamics of pMHC Molecules. Mol Simulat 2008, 34:781-793.

23. Lee $B$, Richards FM: The interpretation of protein structures: estimation of static accessibility. J Mol Biol 1971, 55:379-400.

24. Shrake A, Rupley JA: Environment and exposure to solvent of protein atoms. Lysozyme and insulin. J Mol Biol 1973, 79:351-371.

25. Humphrey W, Dalke A, Schulten K: VMD: visual molecular dynamics. J Mol Graph 1996, 14:33-38. 27-28.

26. Knapp B, Lederer N, Omasits U, Schreiner W: vmdICE: a plug-in for rapid evaluation of molecular dynamics simulations using VMD. J Comput Chem 2010, 31:2868-2873.

27. Sette A, Sidney J, Oseroff C, del Guercio MF, Southwood S, Arrhenius T, Powell MF, Colon SM, Gaeta FC, Grey HM: HLA DR4w4-binding motifs illustrate the biochemical basis of degeneracy and specificity in peptideDR interactions. J Immunol 1993, 151:3163-3170.

28. Baker D, Sali A: Protein structure prediction and structural genomics. Science 2001, 294:93-96.

29. Ehrenmann F, Kaas Q, Lefranc MP: IMGT/3Dstructure-DB and IMGT/ DomainGap Align: a database and a tool for immunoglobulins or antibodies, T cell receptors, MHC, IgSF and MhcSF. Nucleic Acids Res 2010, 38:D301-D307.

30. Chicz RM, Urban RG, Lane WS, Gorga JC, Stern LJ, Vignali DA, Strominger JL: Predominant naturally processed peptides bound to HLA-DR1 are derived from MHC-related molecules and are heterogeneous in size. Nature 1992, 358:764-768.

31. Mutschlechner S, Egger M, Briza P, Wallner M, Lackner $P$, Karle A, Vogt AB, Fischer GF, Bohle B, Ferreira F: Naturally processed T cell-activating peptides of the major birch pollen allergen. J Allergy Clin Immunol 2010, 125:711-718. 718 e1-718 e2.

32. Kropshofer H, Vogt AB, Moldenhauer G, Hammer J, Blum JS, Hammerling GJ: Editing of the HLA-DR-peptide repertoire by HLA-DM. Embo J 1996, 15:6144-6154.

33. Reinherz EL, Tan K, Tang L, Kern P, Liu J, Xiong Y, Hussey RE, Smolyar A, Hare B, Zhang $R$, et al: The crystal structure of a $T$ cell receptor in complex with peptide and MHC class II. Science 1999, 286:1913-1921.

34. Knapp B, Omasits U, Bohle B, Maillere B, Ebner C, Schreiner W, Jahn-Schmid B: 3-Layer-based analysis of peptide-MHC interaction: in silico prediction, peptide binding affinity and T cell activation in a relevant allergenspecific model. Mol Immunol 2009, 46:1839-1844.

35. Knapp B, Omasits U, Schreiner W, Epstein MM: A comparative approach linking molecular dynamics of altered peptide ligands and $\mathrm{MHC}$ with in vivo immune responses. PLoS One 2010, 5:e11653.

\section{Submit your next manuscript to BioMed Central and take full advantage of:}

- Convenient online submission

- Thorough peer review

- No space constraints or color figure charges

- Immediate publication on acceptance

- Inclusion in PubMed, CAS, Scopus and Google Scholar

- Research which is freely available for redistribution

Submit your manuscript at www.biomedcentral.com/submit
C) Biomed Central 